\title{
Le Deeuxième Colloque International sur les genres Niphargus et Gammarus - Lyon, 1973 Compte-rendu de travail pour Niphargus
}

\author{
par \\ R. GINET*
}

En avril 1969 se réunissait, au Museo Civico di Storia Naturale de Vérone, sur invitation du Professeur S. Ruffo, le "Premier Colloque International sur le genre Niphargus". Le but principal de cette réunion était de créer une organisation européenne, de promouvoir une collaboration entre spécialistes, et de proposer un mode de travail unifié, pour tenter de résoudre le difficile problème systématique et taxonomique posé par ce genre d'Amphipodes souterrains. Les diverses et importantes propositions de cette réunion, à laquelle ont participé 16 spécialistes européens, ont été publiées en 1972 sous forme des "Actes du Premier Colloque International sur le genre Niphargus", dans les "Memorie fuori serie n ${ }^{\circ}$ " du Museo Civico di Storia Naturale di Verona.

Par ailleurs, se réunissait en 1971 à Karlsruhe un premier Colloque sur le genre Gammarus.

Quatre ans après Vérone - la deuxième réunion, prévue pour 1971 à Prague, n'ayant pu avoir lieu -, profitant du fait que beaucoup des spécialistes de Niphargus s'intéressent également au genre Gammarus, s'est donc tenu à Lyon un "Deuxième Colloque International sur Gammarus et Niphargus", formé par l'assemblage des participants des deux précédentes réunions. Il a été organisé conjointement par A.L. Roux (Gammarus) et R. Ginet (Niphargus). Ce Colloque lyonnais, qui s'est cléroulé du 9 au 12 juillet 1973 dans les locaux du Département de Biologie Animale et Zoologie de l'Université Claude-Bernard, a réuni une trentaine de participants appartenant à 11 nations différentes.

Nous nous en tiendrons ici uniquement aux travaux concernant le genre hypogé Niphargus.

I. Réunions générales communes aux deux genres d'Amphipodes

Les communications suivantes, ayant Niphargus pour objet, ont été présentées:

- A. Vigna-Taglianti (Rome): Amphipodes souterrains de Turquie.

- A. Skalski (Czestochowa): Variabilité de N. leopoliensis en Pologne.

- N.J. Alouf (Beyrouth): Biogéographie des Niphargus du Liban.

- J. Gibert (Lyon): Essai de systématique biochimique pour Niphargus et Gammarus.

- C. Morand (Lyon): Comparaison biométrique de deux populations de $N$. longicaudatus rhenorhodanensis.

\footnotetext{
* Biologie Souterraine; Université Claude-Bernard, LYON-I. 43, Bd. du 11 novem bre 1918, VILLEURBANNE.
} 
- Projection d'un film (16 mm, couleurs, sonore, 25 minutes) distribué par le S.F.R.S. Paris, réalisé par M.J. Turquin (Lyon, 1973) sur: "Niphargus, un animal cavernicole".

- C. Bou (Moulis): Technique de récolte par pompage des Amphipodes vivant dans les sous-écoulements de cours d'eau.

Ces diverses communications et les commentaires afférents seront exposés en détail dans la revue "Crustaceana".

\section{Réunion du "groupe de Vérone"; systématique de Niphargus}

Parmi les 16 participants au Colloque de Vérone, 7 se sont retrouvés à Lyon: A. Vigna-Taglianti (Rome), J.H. Stock (Amsterdam), A. Skalski (Czestochowa), G. Karaman (Titograd), C. Morand, J. Gibert, R. Ginet (Lyon); ont, en plus, participé au Colloque de Lyon: V. Vader (Tromsoe), C. Bou (Moulis), C. de Broyer (Bruxelles), N.J. Alouf (Beyrouth), J. Mathieu et J.L. Reygrobellet (Lyon). Les organisateurs avaient en outre reçu l'adhésion de collègues étrangers qui, présents à Vérone, n'ont pu pour diverses raisons effectuer le déplacement à Lyon: S. Andreev (Sofia), S. Ruffo (Vérone), B. Sket (Ljubljana), M.Straskraba (Prague), E. Tibaldi (Milan); leur involontaire absence a été vivement regrettée.

Quatre décisions principales avaient été prises au cours du Colloque de Vérone (Allegato 1, 2 et 3, et formation de "groupes d'espèces" avec désignation de responsables); il apparaît, quatre ans après, qu'elles ont été suivies d'effets ou de résultats très inégaux. Les causes en sont diverses; parmi celles qui ont été évoquées à la réunion de Lyon, signalons: l'incertitude qui a longtemps régné pour la réunion d'un second Colloque; le retard dans la publication (excellente par ailleurs) des compte-rendus du Premier Colloque; le manque de liaison entre divers spécialistes; le fait que les travaux de systématique pure sont souvent effectués par les spécialistes en plus de leurs recherches habituelles; la difficulté de réunir des séries de nombreux individus provenant des stations-types; la disparition de certaines de ces stations-types; les problèmes, non encore résolus, concernant la variabilité des populations; etc.

Il importe que, conscience étant prise des difficultés, chacun fasse en sorte d'y rechercher une solution et apporte son active contribution, seuls moyens pour sortir Niphargus de son marasme systématique.

1. Redescription des espèces douteuses ou insuffisamment décrites (Allegato 1 de Vérone):

D'après les renseignements obtenus, le point de ce travail est actuellement le suivant:

$N$. tauri : description terminée, en voie de publication (G. Karaman).

$N$. longicaudatus: description à faire; perspective positive dans les deux années à venir (A. Vigna).

N. tatrensis: description en cours d'achèvement (A. Skalski).

$N$. aquilex: proposition positive pour cette description encore à faire (M. Straskraba). 
N. foreli: difficultés pour retrouver la station-type; travail à faire (S. Ruffo).

$N$. stygius: travail en cours (B. Sket)*.

$N$. elegans: le travail sera entrepris après la redescription de $N$. longicaudatus (A. Vigna)**.

N. puteanus: M. Straskraba doit abandonner ce travail; J.H. Stock se propose pour retrouver les individus typiques et le loco-type de cette espèce, pour une redescription ultérieure.

IL EST INDISPENSABLE que ces divers travaux soient menés à leur bonne fin dans des délais assez brefs; c'est la condition sine qua non pour envisager la clarification du genre Niphargus.

2. Informations :ur les collections disponibles de Niphargus et genres voisins (Allegato 2 de Vérone):

L'essentiel a été fait, dès après le Premier Colloque. Quelques collègues n'ont cependant pas fourni leur liste; il leur est rappelé d'avoir à le faire.

3. Rapports concernant les ouvrages en cours de publication ou en phase d'élaboration, sur le genre Niphargus et genres voisins (Allegato 3 de Vérone):

Cette décision n'a été suivie d'aucune suite positive, par manque de liaison et absence d'informations fournies aux responsables (R. Ginet et D. Dancau). Il apparait cependant très utile que les spécialistes puissent savoir qui travaille, où, et sur quoi, en ce qui concerne la systématique. V. Vader (Tromsoe) propose que des informations lui soient transmises (Musée de Tromsoe, Norvège), pour diffusion par l'intermédiaire de "Amphipods Newsletters", dont il a la responsabilité. Les participants acceptent cette proposition, dont ils remercient son auteur, et demandent à chaque spécialiste de Niphargus de s'y conformer.

\section{Présidence des "groupes d'espèces":}

J.H. Stock signale, en le regrettant, qu'une vingtaine d'espèces nouvelles diverses (groupes kochianus, jovanovici, etc.) ont été décrites depuis 1969 sans que le Président du groupe, désigné à Vérone, n'en ait été saisi; par contre, le processus a bien fonctionné pour d'autres groupes (orcinus). Par ailleurs, M. Straskraba souhaite être déchargé de sa Présidence du groupe elegans-valachicus, qui reste donc sous le contrôle de A. Vigna.

Devant ce résultat inégal, les participants au Colloque de Lyon décident de laisser intacts les groupes et leur Présidence (sauf la modification elegans-valachicus ci-dessus), mais de libérer les membres des groupes, spécialistes reconnus pour leur réputation, de la contrainte d'avoir à en référer aux Présidents des groupes, pour les nouvelles descriptions. Mais il est indispensable qu'un contrôle soit toujours exercé dans le cas de description d'espèces nouvelles par des non-spécialistes du genre Niphargus et des genres voisins, et que, en l'occurrence, ces descriptions soient soumises, préalablement à leur publication, aux Présidents des groupes d'espèces habilités à valider ces taxons nouveaux.

\footnotetext{
* Une révision de $N$. jovanovici en Yougoslavie a été récemment publiée par B. Sket (1972).

** M. Straskraba se propose pour la révision de $N$. valachicus.
} 
La liste des groupes d'espèces et de leurs Présidents est énumérée dans les "Actes du Premier Colloque", page 89.

Il est enfin rappelé que les descriptions ont été codifiées lors du Colloque de Vérone ("Actes", pages 67 à 70) et qu'il est impératif de se conformer à cette codification.

5. Il est enfin souhaité que la prochaine réunion sur la systématique de Niphargus puisse avoir lieu dans un maximum de trois ans, et qu'elle puisse apporter de nombreux éléments positifs à ce problème.

$\mathrm{Au}$ terme du "Deuxième Colloque International Gammarus-Niphargus" il a été proposé que le Colloque suivant aurait lieu, à l'initiative du Dr. M. Meijering, à la Station Limnologique de l'Institut Max-Planck, à SCHLITZ (Allemagne Fédérale).

\section{RESUME}

A la suite d'un Premier Colloque réuni à Vérone en 1969, s'est tenu à Lyon, en juillet 1973, un "Deuxième Colloque International sur le genre Niphargus" (commun avec le genre Gammarus). Au sujet du premier de ces genres, cette réunion a été essentiellement consacrée au bilan des travaux effectués, sur le plan européen, pour la mise au point collective de la systématique, très confuse, de cet Amphipode hypogé. Quelques modifications aux propositions du Colloque de Vérone (publiées en 1972) ont été décidées. Un troisième Colloque, avec le même objectif, se réunira dans un délai maximum de trois ans.

\section{SUMMARY}

Following the First Colloquium held at Verona in 1969, it was planned to hold at Lyon in July, 1973, the Second International Colloquium dealing with the genus Niphargus and also with the genus Gammarus. As subject matter in discussions dealing with the first of these genera, the meeting was devoted mainly the balancing the various viewpoints (based on the European approach) and initiating a collective study of the systematics of these hypogean Amphipoda. The systematics are currently quite confused. Several modifications of the propositions of the Colloquium at Verona (published in 1972) were discussed. A third Colloquium, with the same objectives, is planned after an interval of three years. 